\title{
reviscafuences
}

ISSN: 1575-7072 | e-ISSN: 2172-7775

Páginas: $116-126$

Recibido: 2021-06-23

Revisado: 2021-08-10

Aceptado: 2021-12-31

Preprint: 2022-01-03

Publicación Final: 2022-01-30

www.revistascientificas.us.es/index.php/fuentes/index

DOI: https://doi.org/10.12795/revistafuentes.2022.17471

\section{Diferencias de sexo en habilidades sociales y creatividad en adolescentes: una revisión sistemática}

\section{Sex differences in social skills and creativity in adolescents: a systematic review}

\author{
(D) Alba González Moreno \\ Universidad de Almería (España) \\ D María del Mar Molero Jurado \\ Universidad de Almería (España)
}

\begin{abstract}
Resumen
Las habilidades sociales y la creatividad son un elemento clave en la vida de los jóvenes, debido a que mediante estos dos constructos los adolescentes son capaces de establecer relaciones sociales positivas y solucionar cualquier conflicto que se le interponga. La metodología empleada para esta investigación ha sido de revisión sistemática de la literatura mediante la búsqueda de documentos en las bases de datos Web of Science, Scopus y PsycINFO. Para ello, se han realizado dos búsquedas: una primera para identificar las diferencias de sexo entre adolescentes en relación con las habilidades sociales mediante los descriptores habilidades sociales, género y adolescentes; y otra segunda para conocer dichas diferencias de sexo en cuanto a la creatividad que ha contado con los descriptores creatividad, género y adolescentes. Atendiendo a los resultados cabe destacar en su mayoría los documentos de la muestra señalan que si existen diferencias significativas entre los adolescentes respecto a las habilidades sociales, siendo las chicas quienes mayor nivel de competencias sociales. Por otro lado, referente a la creatividad, la evidencia científica revela que no se encuentran diferencias que permitan distinguir esta capacidad en un sexo u otro. En definitiva, se ha comprobado que las habilidades sociales y la creatividad potencia aspectos positivos en los adolescentes como es un buen rendimiento académico y una alta autoestima, por lo que es imprescindible que las instituciones educativas trabajen estas competencias.
\end{abstract}

\begin{abstract}
Social skills and creativity are a key element in the lives of young people, because through these two constructs adolescents can establish positive social relationships and solving any conflict that comes their way. The methodology used for this research has been a systematic review of the literature by searching for documents in the Web of Science, Scopus and PsycINFO databases. For this, two searches have been carried out: a first to identify the sex differences between adolescents in relation to social skills using the descriptors social skills, gender and adolescents; and a second one to find out these sex differences in terms of creativity that has had the descriptors creativity, gender and adolescents. Considering the results obtained, it should be noted that most of the published studies indicate that there are significant differences between adolescents with respect to social skills, with girls being the ones who have the highest level of these social skills. On the other hand, regarding creativity, scientific evidence reveals that there are no differences that allow distinguishing this ability in one sex or another. In short, it has been proven that social skills and creativity enhance positive aspects in adolescents such as good academic performance and high self-esteem, so it is essential that educational institutions work on these skills.
\end{abstract}

\section{Palabras clave / Keywords}

Creatividad; habilidades sociales; competencias para la vida; socialización; diferencias de género; adolescente; revisión sistemática; investigación.

Creativity; social skills; life skills; socialization; gender differences; adolescent; systematic review; investigation. 


\section{Introducción}

La etapa de la adolescencia abarca un periodo de vulnerabilidad en diversos ámbitos socio-psicológicos que puede afectar a la salud física y mental del propio sujeto dependiendo de la exposición que tenga este a factores psicológicos, biológicos, socioeconómicos, culturales, raciales, étnicos y políticos (Jaimes et al., 2019).

Este ciclo de vida representa un periodo clave, debido a que en estas edades se inician las primeras conductas de riesgo y de protección que van a ser asociadas para la vida adulta (Tajer et al., 2019). Los cambios a nivel físico y socioemocional que se producen durante la adolescencia pueden ocasionar que los individuos de dichas edades experimenten transformaciones sustanciales que afecte a su manera de comportarse (De la Garza y Vásquez, 2015). Algunos de estos cambios hacen referencia al crecimiento físico, la adquisición de madurez sexual y ciertos cambios sociales y emocionales que van a permitir consolidar la propia personalidad del adolescente, así como en la autoconciencia, la autoestima y, en definitiva, su identidad (Viejo y OrtegaRuiz, 2015). El proceso de habituación a estos cambios puede conllevar dificultades en el ajuste psicosocial y un elevado nivel de estrés, lo que puede repercutir de manera negativa en las relaciones afectivas y en el bienestar personal del adolescente (Fernández y Marín, 2018).

Uno de los factores más importantes dentro de la socialización son las habilidades sociales, ya que durante la adolescencia se generan nuevas relaciones sociales y mediante estas capacidades los sujetos son capaces de desenvolverse de manera adecuada con sus iguales y de poder integrarse en nuevos grupos sociales (Suarez-López y Ramos-Noboa, 2018). Las habilidades sociales hacen referencia a todas las conductas y comportamientos que se emplean en la interacción con otras personas y que son necesarias para llevar a cabo relaciones exitosas y afectivas (Esteves et al., 2020). El uso de las habilidades sociales fomenta la autoestima y el bienestar personal, ya que mediante estas competencias sociales los sujetos son capaces de expresar sus opiniones o pensamientos de una manera correcta sin herir los sentimientos de sus receptores, así como, de aceptar las críticas provenientes de otras personas, sin que interfieran en sus propios sentimientos o pensamientos (Gil y Llinás, 2020). Las habilidades sociales potencian otras variables como el rendimiento académico, una alta autoestima y un clima familiar positivo (Núñez et al., 2018; Reis y Gable, 2013).

Los estudios existentes sobre habilidades sociales en la etapa de la adolescencia indican que existen diferencias en relación con el sexo; los hombres tienen menos dificultades a la hora de concertar citas o de efectuar conductas asertivas, mientras que las mujeres son más competentes a la hora de empatizar con otras personas o expresar sentimientos positivos (Holst et al., 2017). Por otro lado, se ha encontrado que las chicas presentan unos niveles más altos de habilidades sociales que los chicos (Betancourth et al., 2017).

Otro de los factores que tienen gran relevancia en la etapa de la adolescencia es la creatividad. La creatividad es el elemento promovedor de que los adolescentes sean capaces de adaptarse a las nuevas circunstancias que se les impone dentro de su vida cotidiana (López-Fernández y Llamas-Salguero, 2018). La creatividad ha sido estudiada desde diferentes enfoques como es el ambiente, el proceso creativo y las características de las personas creativas; por lo que se reconoce la creatividad en diversas acciones humanas (Sastre-Riba y Pascual-Sufrate, 2013). Este constructo se plantea como una característica propia de la mente humana que se encuentra en todas las personas, por lo que mediante los recursos adecuados puede ser enseñada y desarrollada (Taja et al., 2015). Al estar tan presente en el contexto cotidiano este constructo es considerado como una capacidad que se debe de desarrollar en los jóvenes de manera individual con la finalidad de potenciar el desarrollo personal y las habilidades relacionadas con la resolución de problemas y la toma de decisiones en los sujetos (Beghetto, 2013; Ortega et al., 2017).

Atendiendo a las diferencias existentes según el sexo en relación con la creatividad, Belmonte-Lillo y Parodi (2017) señalan que los chicos muestran unas puntuaciones más altas en las variables de pensamiento divergente relacionadas con la creación de nuevas ideas; mientras que las chicas puntúan más alto en el factor de elaboración que se refiere a la capacidad de mejorar o redefinir una idea ya existente. Sin embargo, son muchos los estudios que corroboran que no existen diferencias significativas entre las puntuaciones medias de varones y mujeres en relación con la creatividad independiente de la edad (Bermejo et al., 2014; Elisondo y Donolo, 2011). Por otro lado, contraria a la anterior idea, se ha señalado que si existen diferencias en relación con las capacidades creativas generales; las chicas presentan niveles más altos de creatividad, debido a que llevan a cabo una mayor cantidad de acciones creativas que los chicos (Aranguren e Irazábal, 2012; Chiecher et al., 2018; Elisondo y Donolo, 2016). 


\subsection{Objetivo}

Este trabajo de revisión sistemática tiene como objetivo identificar las diferencias existentes en relación con el sexo en cuanto a la creatividad y a las habilidades sociales en estudiantes adolescentes.

\section{Metodología}

Este trabajo ha sido desarrollado a partir de una metodología basada en una revisión sistemática de la evidencia científica existente. A través de la indagación de las investigaciones presentes se va a ahondar sobre las diferencias existentes en relación con el sexo durante la etapa de la adolescencia acerca de las habilidades sociales y la creatividad y, por tanto, se va a dar respuesta al objetivo planteado. A propósito de realizar una revisión sistemática de calidad se han seguido los principios establecidos por la declaración PRISMA que está diseñada para enriquecer la integridad de los trabajos de revisión (Hutton et al., 2016; Sánchez-Meca, 2010; Urrútia y Bonfin, 2010). Atendiendo a dicha idea, se han aplicado una serie de estadios con el fin de proporcionar validez y calidad a este trabajo de revisión sistemática; estas fases han sido las siguientes: plantear la temática a abordar, proponer el objetivo que se pretende alcanzar, determinar la estrategia de búsqueda planteada, exponer el diagrama de flujo que determina los estudios seleccionados y manifestar los resultados encontrados tras todo este proceso (Alexander, 2020; de la Serna-Tuya et al., 2018; Ramírez et al., 2018).

\subsection{Estrategia de búsqueda}

Las bases de datos que se han utilizado para la realización de esta revisión sistemática han sido Web of Science, Scopus y PsycINFO y como herramienta de apoyo el buscador de Google Académico con la finalidad incluir información adicional sobre la temática a tratar.

Para dar respuesta al objetivo planteado se han realizado dos búsquedas: una, orientada a las habilidades sociales y, la otra, a la creatividad. Ambas búsquedas han contado con una primera indagación con el propósito de conocer los términos empleados en las publicaciones enfocadas a las habilidades sociales o a la creatividad en la etapa de la adolescencia. Una vez realizada esta exploración de la temática a abordar se ha dado comienza a las dos búsquedas:

- Búsqueda 1: La primera búsqueda ha estado focalizada en conocer las diferencias de sexo existentes en relación con las habilidades sociales en los adolescentes. En cuanto a los términos de búsqueda se han tenido en cuenta los descriptores "habilidades sociales", "género" y "adolescentes", ya que las publicaciones existentes sobre esta temática hacen referencia a las diferencias de sexo utilizando el término género y el número de estudios es mayor empleando este concepto. Así pues, la fórmula de búsqueda ha sido realizada mediante la combinación de estos descriptores y el operador booleano AND, dando lugar a la fórmula: habilidades sociales AND género AND adolescentes. No obstante, cabe resaltar que, atendiendo al carácter internacional de las bases de datos escogidas, la búsqueda de estudios ha sido realizada tanto en español como en inglés. Por tanto, la fórmula de búsqueda en inglés ha sido: social skills AND gender AND adolescents.

- Búsqueda 2: La segunda búsqueda, orientada a las diferencias de sexo de los adolescentes en relación con la creatividad, ha sido desarrollada mediante la utilización de los descriptores "creatividad", "género" y "adolescentes". Al igual que ocurría en la búsqueda 1, para hacer referencia a las diferencias de sexo se utiliza el concepto de género $y$, por ello, se ha utilizado este término como descriptor. Así pues, la combinación de los descriptores y el operador booleano AND han generado la fórmula de búsqueda en español: creatividad AND género AND adolescentes; $y$ en inglés: creativity AND gender AND adolescents.

Ambas búsquedas fueron sometidas a filtros de refinamiento con el objetivo de depurar los resultados obtenidos en la búsqueda inicial para así poder atender solo a las investigaciones más relevantes relacionadas sobre, tales como: tipo de documento, idioma, fecha de publicación y accesibilidad al texto completo.

La tabla 1 hace referencia a los aspectos más notables en ambas búsquedas como son las bases de datos, las fórmulas de búsqueda empleadas y los resultados obtenidos. 
Tabla 1

Resultados en cada base de datos según la fórmula de búsqueda

\begin{tabular}{|c|c|c|c|c|}
\hline Búsquedas & Base de datos & Idioma & Fórmulas de búsqueda & $\begin{array}{l}\text { № de resultados } \\
\text { tras aplicar filtros }\end{array}$ \\
\hline \multirow{6}{*}{ Búsqueda 1} & \multirow[b]{2}{*}{ Web of Science } & Español & $\begin{array}{l}\text { habilidades sociales AND } \\
\text { género AND adolescentes }\end{array}$ & 8 \\
\hline & & Inglés & $\begin{array}{r}\text { social skills AND gender } \\
\text { AND adolescents }\end{array}$ & 538 \\
\hline & \multirow{2}{*}{ Scopus } & Español & $\begin{array}{l}\text { habilidades sociales AND } \\
\text { género AND adolescentes }\end{array}$ & 1 \\
\hline & & Inglés & $\begin{array}{r}\text { social skills AND gender } \\
\text { AND adolescents }\end{array}$ & 339 \\
\hline & \multirow{2}{*}{ PsyclNFO } & Español & $\begin{array}{l}\text { habilidades sociales AND } \\
\text { género AND adolescentes }\end{array}$ & 4 \\
\hline & & Inglés & $\begin{array}{r}\text { social skills AND gender } \\
\text { AND adolescents }\end{array}$ & 581 \\
\hline \multirow{6}{*}{ Búsqueda 2} & \multirow{2}{*}{ Web of Science } & Español & $\begin{array}{r}\text { creatividad AND género } \\
\text { AND adolescentes }\end{array}$ & 0 \\
\hline & & Inglés & $\begin{array}{r}\text { creativity AND gender AND } \\
\text { adolescents }\end{array}$ & 25 \\
\hline & \multirow{2}{*}{ Scopus } & Español & $\begin{array}{r}\text { creatividad AND género } \\
\text { AND adolescentes }\end{array}$ & 0 \\
\hline & & Inglés & $\begin{array}{r}\text { creativity AND gender AND } \\
\text { adolescents }\end{array}$ & 20 \\
\hline & \multirow{2}{*}{ PsycINFO } & Español & $\begin{array}{r}\text { creatividad AND género } \\
\text { AND adolescentes }\end{array}$ & 0 \\
\hline & & Inglés & $\begin{array}{r}\text { creativity AND gender AND } \\
\text { adolescents }\end{array}$ & 47 \\
\hline
\end{tabular}

\subsection{Criterios de elegibilidad}

El recurso que se ha utilizado para establecer tanto los criterios de inclusión como los de exclusión ha sido la conocida como estrategia PICoS (Landa-Ramírez y Arredondo-Pantaleón, 2014; Richardson et al., 1995). Esta herramienta se caracteriza por establecer los criterios de elegibilidad a partir de sus cuatro variables: sujetos, temática de interés, contexto y diseño del estudio.

- Sujetos: Se han seleccionado todos aquellos estudios provenientes de una población adolescente, es decir, de entre 12 a 18 años de edad aproximadamente y que no presentasen ningún tipo de problema ya sea de carácter físico o mental. Por tanto, se han descartado todas aquellas investigaciones cuya muestra correspondía a otra etapa evolutiva o que, a pesar de ser adolescentes, presentaban algún tipo de trastorno o necesidad educativa.

- Temática de interés: En vista de que la temática a abordar hace referencia a las diferencias de sexo en dos variables: habilidades sociales y creatividad, se han seleccionado las investigaciones que incluían datos sobre las disimilitudes existentes entre chicos y chicas en relación a algunas de dichos constructos. Por esta razón, se han desestimado aquellos estudios que no daban información relevante sobre las diferencias de sexo entre adolescentes en habilidades sociales o en creatividad.

- Contexto: Las investigaciones incluidas se caracterizan por estar desarrolladas en un contexto educativo, por ello se han desvinculado de este trabajo de revisión sistemática aquellos artículos que hacen referencia a un ámbito clínico o familiar.

- Diseño del estudio: Este trabajo de revisión de la literatura ha contemplado todos los estudios indexados en revistas científicas y de carácter cuantitativo publicados en la última década (20212011) en español o en inglés y que tuviera disponible su texto completo. Por consiguiente, se han declinado los estudios procedentes de libros, capítulos de libro o trabajos de fin de estudios, así como, aquellos cuyo idioma es distinto al español o ingles (ruso, francés, chino, etc.), que estaban publicados antes del año 2011, que hacían referencia a trabajos de revisión o de carácter cualitativo o que no se podía acceder al texto completo. 


\subsection{Proceso de selección de las publicaciones}

Una vez establecidos los criterios de elegibilidad, se han tenido en cuenta dichos criterios con la finalidad de abarcar solo aquellos estudios que dieran respuesta al objetivo planteado.

Por un lado, la búsqueda 1 (Figura 1A) ha dado lugar a un total de 4447 documentos que, una vez descartados los estudios que no se encontraban dentro de los criterios de inclusión, se ha reducido dicha selección a 15 trabajos. Por otro lado, la búsqueda 2 (Figura 1B), al tratarse de la creatividad que es un concepto menos estudiado, se ha obtenido un total de 370 resultados en la búsqueda previa. Una vez desestimados los estudios que no cumplían con los requisitos para poder ser integrados en este trabajo de revisión sistemática por diferentes motivos como la fecha de publicación, el idioma o que no daba respuesta al objetivo propuesto, se ha contado con un total 8 de artículos para esta última búsqueda. Todo este proceso se muestra reflejado mediante la figura 1.

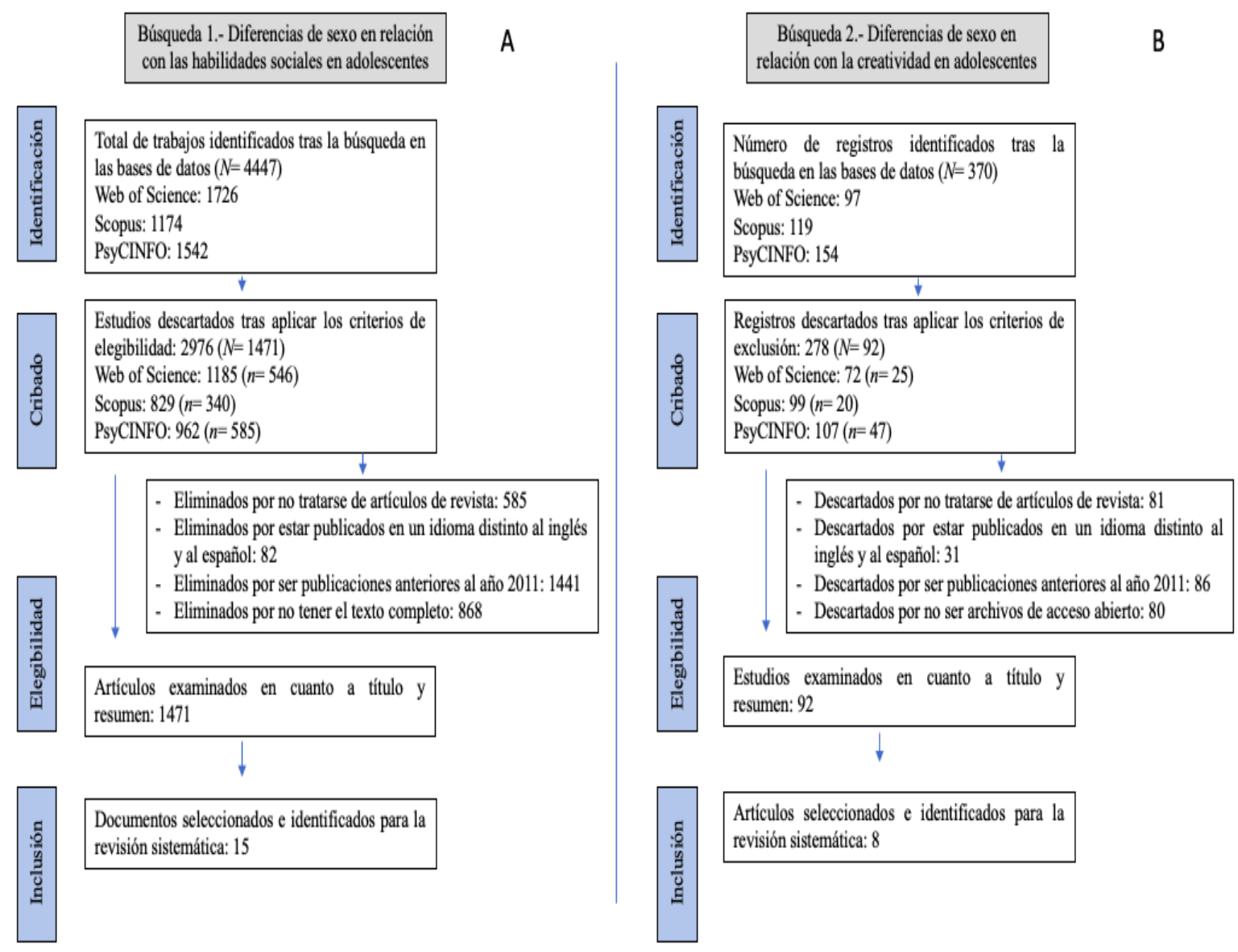

Figura 1. Diagrama de flujo del proceso de selección de los trabajos

\section{Resultados}

Una vez implantados los criterios de inclusión y exclusión, descartando así los estudios que no contaban con dichos criterios, se ha realizado una revisión manual en cuanto a su título, resumen y contenido en ambas búsquedas con la finalidad de comprobar cuáles son las investigaciones más idóneas para su inclusión en esta revisión sistemática. La búsqueda 1, orientada a las diferencias de sexo en relación con las habilidades sociales en la etapa de la adolescencia, ha obtenido un total de 15 estudios; la búsqueda 2, enfocada a las diferencias existentes en el sexo en cuanto a la creatividad en los adolescentes, ha concluido con la selección de 8 artículos. Así pues, entre ambas búsquedas se ha recabado un total de 23 investigaciones. 
Para comenzar, la tabla 2 muestra algunos de los aspectos más relevantes de la búsqueda 1 . Dentro de dichos aspectos se expone: el autor/es y fecha de publicación del artículo; el país de procedencia; la muestra que se ha utilizado para la elaboración del trabajo; y las diferencias existentes en cuanto al sexo, indicando así si son las chicas o los chicos quienes presentan una mayor puntuación en las habilidades sociales o si, por el contrario, no existen diferencias significativas entre ambos sexos.

\section{Tabla 2}

Características de los estudios seleccionados

\begin{tabular}{|c|c|c|c|c|}
\hline \multirow[t]{2}{*}{ Autor/es y año } & \multirow[t]{2}{*}{ País } & \multirow[t]{2}{*}{ Muestra } & \multicolumn{2}{|c|}{$\begin{array}{c}\text { Diferencias por sexo en relación a las } \\
\text { HH.SS. }\end{array}$} \\
\hline & & & q & $0^{\lambda}$ \\
\hline Portela-Pino et al. (2021) & España & $\begin{array}{c}N=964 \\
(11 \text { a } 18 \text { años })\end{array}$ & $=$ & $=$ \\
\hline $\begin{array}{l}\text { Sánchez-Hernando et al. } \\
\text { (2021) }\end{array}$ & España & $\begin{array}{c}N=1745 \\
(12 \text { a } 14 \text { años })\end{array}$ & $\uparrow$ & $\downarrow$ \\
\hline Tacca et al. (2020) & Perú & $\begin{array}{c}N=324 \\
(16 \text { a } 18 \text { años })\end{array}$ & $\downarrow$ & $\uparrow$ \\
\hline Andrade et al. (2020) & Colombia & $\begin{array}{c}N=400 \\
(12 \text { a } 18 \text { años })\end{array}$ & $\uparrow$ & $\downarrow$ \\
\hline Sosa y Salas-Blas (2020) & Perú & $\begin{array}{c}N=212 \\
(11 \text { a } 20 \text { años })\end{array}$ & $\uparrow$ & $\downarrow$ \\
\hline Schenk et al. (2020) & Países Bajos & $\begin{array}{c}N=596 \\
(12 \text { a } 14 \text { años })\end{array}$ & $=$ & $=$ \\
\hline Salavera et al. (2019) & España & $\begin{array}{c}N=1358 \\
(12 \text { a } 17 \text { años })\end{array}$ & $\uparrow$ & $\downarrow$ \\
\hline $\begin{array}{l}\text { Akelaitis y Lisinskiene } \\
(2018)\end{array}$ & Lituania & $\begin{array}{c}N=468 \\
(15 \text { y } 16 \text { años })\end{array}$ & $\uparrow$ & $\downarrow$ \\
\hline Aydos y Akyol (2018) & Turquía & $\begin{array}{c}N=383 \\
(12 \text { a } 14 \text { años })\end{array}$ & $\uparrow$ & $\downarrow$ \\
\hline $\begin{array}{l}\text { González y } \quad \text { Ramirez } \\
(2017)\end{array}$ & México & $N=557$ & $=$ & $=$ \\
\hline Zegarra y Cuba (2017) & Perú & $\begin{array}{c}N=179 \\
(10 \text { a } 19 \text { años })\end{array}$ & $\uparrow$ & $\downarrow$ \\
\hline $\begin{array}{l}\text { Pulido-Acosta y Herrera- } \\
\text { Clavero (2016) }\end{array}$ & España & $\begin{array}{c}N=557 \\
(12 \text { a } 18 \text { años })\end{array}$ & $\downarrow$ & $\uparrow$ \\
\hline Vorobjov et al. (2014) & Estonia & $\begin{array}{c}N=2460 \\
(15 \text { a } 16 \text { años })\end{array}$ & $\uparrow$ & $\downarrow$ \\
\hline $\begin{array}{l}\text { Von Hohendorff et al. } \\
(2013)\end{array}$ & Brasil & $\begin{array}{c}N=50 \\
(12 \text { a } 17 \text { años })\end{array}$ & $=$ & $=$ \\
\hline Cardozo et al. (2011) & Argentina & $\begin{array}{c}N=124 \\
(13 \text { a } 18 \text { años })\end{array}$ & $\uparrow$ & $\downarrow$ \\
\hline
\end{tabular}

Nota: $\rho$ indica sexo femenino; $\widehat{o}$ indica sexo masculino; = hace referencia a similitud en niveles de habilidades sociales; $\uparrow$ significa mayor nivel de habilidades sociales; $\downarrow$ implica menor nivel de habilidades sociales.

Uno de los aspectos más destacados es que existe una opinión unánime sobre cuál es el sexo qué mayor puntuación obtiene en habilidades sociales, ya que 9 de los 15 estudios seleccionados indican que en la etapa de la adolescencia son las chicas quienes obtienen niveles más elevados de habilidades sociales en comparación con los chicos de su misma edad. Esta idea es corroborada por Sánchez-Hernando et al. (2020), quienes señalan que aunque todos los miembros que participaron en su estudio tienen unas buenas habilidades sociales, más niñas que niños alcanzaron un nivel más alto; algunas de las diferencias más claras es que los chicos puntúan mas alto en conocer gente nueva y por hacer pensar a los demás que sus ideas son mejores que las de los demás, mientras que las chicas prestan más atención a las explicaciones que se les ofrecen y tienen interacciones más positivas con otras personas. Andrade et al. (2020) muestran que de sus participantes cerca del $70 \%$ de las chicas tienen un buen nivel de habilidades sociales, pero que no existen diferencias de sexo en relación a los conflictos primarios aunque si que las chicas tienen mayores problemas de autoestima y que los chicos sufren una mayor adicción a los videojuegos; datos que también se incluyen dentro de los estudios de Zegarra y Cuba (2017) y Vorobjov et al. (2014) quienes indican que los chicos presentan menores habilidades sociales y un mayor índice de adicción ya sea en aparatos electrónicos o en sustancias psicoactivas. Por otro lado, el estudio de Sosa y Salas-Blas (2020) revela que las habilidades 
sociales se encuentran inmersas en la mayoría de los adolescentes sobre todo las habilidades relacionadas con la comunicación, pero que se encuentran diferencias estadísticamente significativas en las habilidades de planificación, donde las chicas puntúan más alto. La investigación de Salavera et al. (2019) centrada en conocer como se relacionan las variables de habilidades sociales, inteligencia emocional e internalización de problemas, expone que las chicas muestran mayores puntuaciones tanto en habilidades sociales como inteligencia emocional; variables que se relacionan de manera inversa con la internalización de los problemas. Asimismo, Akelaitis y Lisinskiene (2018) revelan que en la comparación de habilidades socioemocionales respecto al género, las chicas son más capaces de comprender emociones y de colaborar con otras personas y, además, reportan mayor participación en los aspectos emociones y llevan a cabo más comportamientos prosociales que los niños. Al igual ocurre con el estudio de Aydos y Akyol (2018), quienes argumentan que los chicos adolescentes tienen menores habilidades de comunicación, de resolución de problemas, de aprendizaje socioemocional y son menos capaces de afrontar el estrés que las chicas. Por último, el trabajo de Cardozo et al. (2011) señala que si existen diferencias significativas entre habilidades para la vida y el sexo, siendo las chicas quienes mayor puntuación alcanzan en las variables de ansiedad-timidez, empatía, autoconcepto social y conducta de sumisión.

No obstante, aunque el número de estudios que lo afirman es menor, también hay investigadores que señalan que no existen diferencias sobre las habilidades sociales entre los adolescentes (Schenk et al., 2020; Von Hohendorff et al., 2013). La mayoría de las variables relativas a las competencias socioemocionales como es la autoconciencia, conciencia social o toma de decisiones no obtuvieron ninguna diferencia significativa entre chicos y chicas, pero si hubo diferencias en la autogestión siendo mayor en los chicos y en el manejo de las relaciones, la cual fue mayor en las chicas (Portela-Pino et al., 2020). Al igual ocurre con los estudios que indican que los chicos presentan mayores puntuaciones de habilidades sociales que las chicas como es el caso de Tacca et al. (2020), donde se establece que los chicos además de un mayor nivel de habilidades sociales también presentan una puntuación más alta en autoconcepto y autoestima. El estudio de PulidoAcosta y Herrera-Clavero (2016) también indica que los chicos presentan mayores habilidades sociales que las chicas y que esta variable es inversamente proporcional con la variable miedo, por lo que las chicas al tener menor niveles de habilidades sociales presentan niveles superiores de miedo.

\section{Tabla 2}

Características de los estudios seleccionados

\begin{tabular}{|c|c|c|c|c|}
\hline \multirow[t]{2}{*}{ Autor/es y año } & \multirow[t]{2}{*}{ País } & \multirow[t]{2}{*}{ Muestra } & \multicolumn{2}{|c|}{$\begin{array}{c}\text { Diferencias por sexo en relación a las } \\
\text { HH.SS. }\end{array}$} \\
\hline & & & 웅 & $\hat{0}$ \\
\hline $\begin{array}{l}\text { Sánchez-Escobedo et } \\
\text { al. (2021) }\end{array}$ & Lituania & $\begin{array}{c}N=354 \\
(16 \text { a } 19 \text { años })\end{array}$ & $=$ & $=$ \\
\hline Azevedo et al. (2019) & Portugal & $\begin{array}{c}N=131 \\
(12 \text { a } 15 \text { años })\end{array}$ & $=$ & $=$ \\
\hline Sali (2019) & Turquía & $\begin{array}{c}N=145 \\
(15 \text { a } 18 \text { años })\end{array}$ & $=$ & $=$ \\
\hline $\begin{array}{l}\text { Belmonte-Lillo y Parodi } \\
\text { (2017) }\end{array}$ & España & $\begin{array}{c}N=670 \\
(12 \text { a } 16 \text { años })\end{array}$ & $\uparrow$ & $\downarrow$ \\
\hline Amado et al. (2017) & México & $\begin{array}{c}N=922 \\
(11 \text { a } 17 \text { años })\end{array}$ & $\uparrow$ & $\downarrow$ \\
\hline Mefoh et al. (2017) & Nigeria & $\begin{array}{c}N=240 \\
(15 \text { a } 17 \text { años })\end{array}$ & $\downarrow$ & $\uparrow$ \\
\hline Bart et al. (2015) & Minnesota & $\begin{array}{c}N=996 \\
(13 \text { a } 17 \text { años })\end{array}$ & $=$ & $=$ \\
\hline Brand et al. (2011) & Suiza & $\begin{array}{c}N=5580 \\
(17 \text { a } 19 \text { años })\end{array}$ & $\uparrow$ & $\downarrow$ \\
\hline
\end{tabular}

Nota: $q$ indica sexo femenino; $\delta$ indica sexo masculino; = hace referencia a similitud en niveles de habilidades sociales; $\uparrow$ significa mayor nivel de habilidades sociales; $\downarrow$ implica menor nivel de habilidades sociales.

Atendiendo a la búsqueda 2, orientada en conocer las diferencias de sexo existentes en relación con la creatividad entre los adolescentes, se ha elaborado la tabla 3. Esta tabla reporta los aspectos más relevantes de los estudios que tratan sobre esta temática como son: autor y año de publicación; país donde se ha ejecutado la investigación; muestra de participantes que han intervenido en los estudios; y, las diferencias 
encontradas por sexo que, al igual que en la tabla anterior, se indica si son las chicas o los chicos quienes mayores porcentajes de creatividad poseen o si no existen diferencias entre ambos sexos.

La mayoría de los resultados encontrados en esta búsqueda indican que no existen diferencias entre chicos y chicas en relación con la creatividad en la etapa de la adolescencia. Tal y como señalan Sánchez-Escobedo et al. (2021), no existen diferencias significativas entre chicos y chicas, pero en su estudio los chicos puntúan más alto en originalidad para inventar tareas y las chicas en tareas visoespaciales. Al igual ocurre con el estudio de Azevedo et al. (2019), aunque no las puntuaciones no son muy dispares para establecer diferencias de sexo, si que los chicos tienden a puntuar más alto en originalidad y las chicas en fluidez. Al contrario ocurre en el trabajo de Sali (2019) y Bart (2015) que, aunque no se observaron diferencias significativas en las puntuaciones de fluidez, originalidad y elaboración entre ambos sexos, si que los adolescentes varones obtuvieron unas medias algo más altas en fluidez.

Por otro lado, otras investigaciones si que han encontrado diferencias de sexo en relación con la creatividad donde se establecen que las chicas poseen niveles más altos de creatividad que los chicos. Las chicas tienden a puntuar más alto en el factor de elaboración (Belmonte-Lillo y Parodi, 2017) y en otras variables que se relacionan con la creatividad como es la percepción de las necesidades psicológicas básicas de apoyo, la motivación intrínseca, esfuerzo y comportamiento positivo (Amado et al., 2017); Brand et al. (2011) señala que las mujeres adolescentes se describen a sí mismas como más creativas y, en su estudio, relaciona un nivel alto de creatividad con problemas de sueño y estrés.

Para finalizar cabe resaltar el trabajo de Mefoh et al. (2017), el cual es el único que se ha recogido que indica que los chicos tienen una capacidad mayor en la resolución de problemas que las chicas.

\section{Discusión y conclusiones}

La adolescencia es una etapa de la vida del ser humano que abarca una gran diversidad de cambios, tanto a nivel físico como emocional, y que mediante estos los sujetos pueden verse afectados en su salud mental o incluso iniciarse en ciertas conductas de riesgo como es el consumo de sustancias (De la Garza y Vásquez, 2015; Jaimes et al., 2019; Tajer et al., 2019). Algunos de los cambios más representativos en este periodo son el crecimiento físico, la madurez sexual y cambios emocionales como la autoestima que pueden repercutir, tanto positivamente como negativamente, en las relaciones interpersonales que se desarrollan entre iguales o con otros miembros (Fernández y Marín, 2018; Viejo y Ortega-Ruiz, 2015).

Las habilidades sociales y la creatividad son dos variables que repercuten en la socialización de los jóvenes que, durante la adolescencia, es uno de los factores más importantes. En primer lugar, mediante las habilidades sociales se hace referencia a todas aquellas aptitudes y comportamientos que se llevan a cabo con la finalidad de establecer relaciones sociales de calidad; desarrollando así un bienestar personal idóneo que potencia un buen rendimiento escolar, un clima familiar positivo y una alta autoestima (Esteves et al., 2020; Gil y Llinás, 2020; Núñez et al., 2018; Reis y Gable, 2013; Suarez-López y Ramos-Noboa, 2018).

Atendiendo a los resultados encontrados, es preciso destacar como la mayoría de la evidencia científica indica que existen diferencias entre chicos y chicas adolescentes en relación con las habilidades sociales. Se manifiesta que todos los adolescentes disponen de habilidades sociales en mayor o menos medida, pero que las chicas tienden a puntuar más alto que los chicos en tener relaciones positivas, comprender emociones, habilidades relacionadas con la planificación y mayores niveles de inteligencia emocional y empatía (Akelaitis y Lisinskiene; 2018; Betancourrth et al., 2017; Cardozo et al., 2011; Holst et al., 2017; Salavera et al., 2019; Sánchez-Hernando et al., 2020; Sosa y Salas-Blas, 2020).

La idea de que los que los chicos tengan menores niveles de habilidades sociales se relaciona con que los adolescentes varones tienen mayores problemas de adición y de aprendizaje socioemocional que las chicas (Andrade et al., 2020; Aydis y Akyol, 2018; Vorobjov et al., 2014; Zegarra y Cuba, 2017). No obstante, otros estudios señalan que no existen diferencias en relación al sexo en cuanto a las habilidades sociales, ya que se han obtenido puntuaciones similares en las dimensiones como la autoconciencia, conciencia social o toma de decisiones (Portela-Pino et al., 2020; Schenk et al., 2020; Von Hohendorff et al., 2013).

Por otro lado, aunque en menor medida, también hay investigaciones que reflejan que los chicos tienen unos niveles de habilidades sociales más altos que las chicas como es el caso de los estudios de Pulido-Acosta y Herrera Clavero (2016) y Tacca et al. (2020), quienes indican que el sexo masculino abarca mayores niveles de autoconcepto, autoestima y menor sensación de miedo.

Por otro lado, haciendo referencia a la creatividad, este constructo propio de la mente humana promueve que las personas, en este caso los adolescentes, sean capaces de adaptarse a todos los acontecimientos que se impongan dentro de su vida cotidiana y resolver sus problemas mediante los recursos adecuados (Beghetto, 
2013; López-Fernández y Llamas-Ortega et al., 2017; Salguero, 2018; Taja et al., 2015); y puede ser entendida desde diferentes enfoques como una característica propia del ser humano, como algo que se encuentra en el ambiente o como un proceso mental (Sastre-Riba y Pascual-Sufrate, 2013). Atendiendo a los resultados encontrados cabe destacar la disparidad de estos, aunque un porcentaje mayor estima que no existen diferencias significativas de sexo en la creatividad entre adolescentes (Azevedo et al., 2019; Bart et al., 2015; Bermejo et al., 2014; Elisondo y Donolo, 2011; Sali, 2019; Sánchez-Escobedo et al., 2021); pero si que las chicas tienden a puntuar algo más alto en fluidez y los chicos en originalidad. Por otro lado, otros estudios, concretamente los de Amado et al. (2017), Belmonte-Lillo y Parodi (2017) y Brand et al. (2011) si estiman que existan diferencias, siendo las chicas quienes mayores niveles de creatividad poseen sobre todo a la hora de mejorar o redefinir una idea ya existente; este juicio ha sido corroborado por otras investigaciones (Aranguren e Irazábal, 2012; Chiecher et al., 2018; Elisondo y Donolo, 2016). Para finalizar, el trabajo de Mehfon et al. (2017) es contrario a dicha idea y muestra que los chicos tienen una mayor capacidad creativa a la hora de establecer soluciones originales a un determinado conflicto.

Para concluir, cabe destacar como las habilidades sociales y la creatividad son un elemento clave dentro de la vida de los adolescentes que potencian la resolución de conflictos y el bienestar personal. Atendiendo a las limitaciones cabe resaltar como este trabajo de revisión sistemática ha estado compuesto por dos búsquedas bibliográficas, teniendo que ser adaptadas estas búsquedas que fueran lo más similares posibles. Además, cabe resaltar como la literatura científica sobre la creatividad en la etapa de la adolescencia es escasa, lo cual ha dado un menor número de resultados que la búsqueda sobre habilidades sociales. Como futuras líneas de investigación puede resultar interesante integrar otras variables que son relevantes en el desarrollo personal de una etapa tan icónica como es la adolescencia tales como la inteligencia emocional o la autoestima. Haciendo hincapié en las implicaciones prácticas que puede tener esta revisión sistemática es preciso señalar como, a partir de este trabajo, se pueden identificar si existen o no diferencias de sexo en relación tanto de la creatividad como de las habilidades sociales entre los adolescentes y conocer las diversas similitudes encontradas sobre los estudios recogidos.

En definitiva, se ha encontrado que durante la etapa de la adolescencia en relación con las habilidades sociales si que existen diferencias, siendo pues las chicas quienes mayores niveles tienen de estas capacidades; por el contrario, en la creatividad, la mayoría de los resultados obtenidos indican que no existen diferencias significativas entre ambos sexos. Es preciso resaltar como las habilidades sociales y la creatividad potencian la calidad de vida de los adolescentes y, por ello, es necesario que estas aptitudes se trabajen dentro de las escuelas con la finalidad de desarrollar individuos capaces de establecer relaciones interpersonales positivas.

\section{Agradecimientos}

El presente trabajo cuenta con el apoyo del Ministerio de Educación y Formación Profesional a través del programa de ayudas para la Formación de Profesorado Universitario (FPU) otorgado a Alba González Moreno con referencia FPU19/01570.

\section{Referencias}

Akelaitis, A.V., \& Lisinskiene, A.R. (2018). Social Emotional Skills and Prosocial Behaviour among 15-16-year-old Adolescents. European Journal of Contemporary Education, 7(1), 21-28. http://doi.org/10.13187/ejced.2018.1.21 Alexander, P.A. (2020). Methodological guidance paper: The art and science of quality systematic reviews. Review of Educational Research, 90(1), 6-23. https://doi.org/10.3102\%2F0034654319854352

Amado, D., Sánchez, P.A., \& Molero, P. (2017). Creativity associated with the application of a motivational intervention programme for the teaching of dance at school and its effect on both genders. PLoS ONE, $12(3), 14$. http://dx.doi.org/10.1371/journal.pone.0174393

Andrade, J., Mendoza, M., Zapata, J., \& Sierra, L. (2020). Relación entre conflictos de la adolescencia y habilidades sociales en adolescentes de una Institución Educativa de Risaralda. Pensamiento Americano, 13(25), 52-61. http://dx.doi.org/10.21803/pensam.13.25.385

Aranguren, M., \& Irazábal, N. (2012). Diseño de una escala para la evaluación del comportamiento creativo en diferentes dominios. Ciencias Psicológicas, 6(1), 29-41. https://doi.org/10.22235/cp.v6i1.60

Aydos, S., \& Akyol, A. (2018). The Effect of Different Weight Status on Social-Emotional Skills of Adolescents. International Journal of Child Health and Nutrition, 7(4), 184-193. https://doi.org/10.6000/1929-4247.2018.07.04.8

Azevedo, I., Morais, M. F., \& Martins, F. (2019). The future problem-solving program international: An intervention to promote creative skills in Portuguese adolescents. The Journal of Creative Behavior, 53(3), $263-273$. http://dx.doi.org/10.1002/jocb.175 
Bart, W. M., Hokanson, B., Sahin, I., \& Abdelsamea, M. A. (2015). An investigation of the gender differences in creative thinking abilities among 8th and 11th grade students. Thinking Skills and Creativity, 17, 17-24. http://dx.doi.org/10.1016/i.tsc.2015.03.003

Beghetto, R.A. (2013). Killing Ideas Softly? The Promise and Perils of Creativity in the Classroom. Information Age Publishing, Inc.

Belmonte-Lillo, V.M., \& Parodi, A.I. (2017). Creatividad y adolescencia: Diferencias según género, curso y nivel cognitivo. European Journal of Investigation in Health, Psychology and Education, 7(3), $177-188$. https://doi.org/10.30552/ejihpe.v7i3.205

Bermejo, R., Ferrando, M.M., Sainz, M., Soto, G., \& Ruiz, M.J. (2014). Procesos cognitivos de la creatividad en estudiantes universitarios. Educatio Siglo XXI, 32(2), 41-58. https://doi.org/10.6018///202151

Betancourth, S., Zambrano, C., Ceballos, A., Benavides, V., \& Villota, N. (2017). Habilidades sociales relacionadas con el proceso de comunicación en una muestra de adolescentes. Revista Psicoespacios, 11(18), $133-148$. https://doi.org/10.25057/21452776.898

Brand, S., Beck, J., Kalak, N., Gerber, M., Kirov, R., Pühse, U., Hatzinger, M., \& Holsboer-Trachsler, E. (2011). Dream recall and its relationship to sleep, perceived stress, and creativity among adolescents. Journal of Adolescent Health, 49(5), 525-531. http://dx.doi.org/10.1016/i.jadohealth.2011.04.004

Cardozo, G., Dubini, P., Fantino, I., \& Ardiles, R. (2011). Habilidades para la vida en adolescentes: diferencias de género, correlaciones entre habilidades y variables predictoras de la empatía. Psicología desde el Caribe, 28, $107-132$. https://bit.ly/3zMH8uu

Chiecher, A.C., Elisondo, R.C., Paoloni, P.V., \& Donolo, D.S. (2018). Creatividad, género y rendimiento académico en ingresantes de ingeniería. Revista Iberoamericana de Educación Superior, 24, $138-151$. http://dx.doi.org/10.22201/iisue.20072872e.2018.24.266

De la Garza, Á.L., \& Vásquez, N.B. (2015). Programa de intervención para favorecer el desarrollo integral en adolescentes de secundaria. Revista de Psicología, Procesos Psicológicos y Sociales, 11(1), 1-37. https://bit.ly/3zP6g3D

De la Serna-Tuya, A.S., González-Calleros, J.M., \& Navarro, Y. (2018). Las Tecnológicas de Información y Comunicación en el preescolar: Una revisión bibliográfica. Campus Virtuales, 7(1), 19-31. https://bit.ly/3gR4VBS

Elisondo, R.C., \& Donolo, D.S. (2011). Los estímulos en un test de creatividad. Incidencias según género, edad y escolaridad. Boletín de Psicología, 101, 51-65. https://bit.ly/3d6YUPh

Elisondo, R.C., \& Donolo, D.S. (2016). Construcción y análisis de las propiedades psicométricas del Cuestionario de Acciones Creativas en población argentina. Psiencia. Revista Latinoamericana de Ciencia Psicológica, 8(1), 1-21 http://doi.org/10.5872/psiencia/8.1.42

Esteves, A.R., Paredes, R.P., Calcina, C.R., \& Yapuchura, C.R. (2020). Habilidades Sociales en adolescentes y Funcionalidad Familiar. Comuni@cción, 11(1), 16-27. https://dx.doi.org/10.33595/2226-1478.11.1.392

Fernández, M.J., \& Marín, V. (2018). La educación para la salud en la adolescencia temprana para afrontar los cambios físicos y emocionales. Revista Enfermería Actual en Costa Rica, 1(Edición Especial), 1-11. https://bit.ly/3qk8fbQ

Gil, S., \& Llinás, A. (2020). Grandes herramientas para pequeños guerreros. Habilidades sociales. Editorial Flamboyant.

González, B., \& Ramírez, V. (2017). Acoso escolar y habilidades sociales en estudiantes de educación básica. Ciencia ergo-sum, 24(2), 109-116. https://doi.org/10.30878/ces.v24n2a2

Holst, I.C., Galicia, B.Y, Gómez, V.G., \& Degante, G.A. (2017). Las habilidades sociales y sus diferencias en estudiantes universitarios. Revista Especializada en Ciencias de la Salud, 20(2), 22-29. https://bit.ly/3zLbT37

Hutton, B., Catalá-López, F., \& Moher, D. (2016). La extensión de la declaración PRISMA para revisiones sietemáticas que incorporan metaanálisis en red: PRISMA-NMA. Medicina Clínica, 147(6), $262-266$. http://doi.org/10.1016/i.medcli.2016.02.025

Jaimes, C., Cruz, L., Vega, E., Balladares, P., \& Matta, H. (2019). Habilidades sociales en estudiantes adolescentes de un centro preuniversitario de Lima Norte. Health Care \& Global Health, 3(1), 39-43. https://doi.org/10.22258/hgh.2019.31.54

Landa-Ramírez, E., \& Arredondo-Pantaleón, A.J. (2014). Herramienta pico para la formulación y búsqueda de preguntas clínicamente relevantes en la psicooncología basada en la evidencia. Psicooncología, 11(2/3), $259-270$. http://doi.org/10.5209/rev psic.2014.v11.n2-3.47387

López-Fernández, V., \& Llamas-Salguero, F. (2018). Neuropsicología del proceso creativo. Un enfoque educativo. Revista Complutense de Educación, 29(1), 113-127. https://doi.org/10.5209/RCED.52103

Mefoh, P. C., Nwoke, M. B., Chukwuorji, J. C., \& Chijioke, A. O. (2017). Effect of cognitive style and gender on adolescents' problem-solving ability. Thinking Skills and Creativity, 25, 47-52. http://dx.doi.org/10.1016/j.tsc.2017.03.002

Núñez, C., Hernández, V., Jerez, D., Rivera, D., \& Núñez, M. (2018). Las habilidades sociales en el rendimiento académico en adolescentes. Revista de Comunicación de la SEECl,47, 37-49. http://doi.org/10.15198/seeci.2018.47.37-49

Ortega, M.A., Llamas, F., \& López-Fernández, V. (2017). Efecto de un programa de enseñanza creativa en las inteligencias múltiples y la creatividad en alumnos de 3 años. Revista de Currículum y Formación del Profesorado, 21(2), 67-83. https://doi.org/10.30827/profesorado.v21i2.10325

Portela-Pino, I., Alvariñas-Villaverde, M., \& Pino-Juste, M. (2021). Habilidades socioemocionales en la adolescencia. Influencia de variables personales y extraescolares. Revista Internacional de Investigación Ambiental y Salud Pública, 18(9), 4811. http://dx.doi.org/10.3390/ijerph18094811 
Pulido-Acosta, F., \& Herrera-Clavero, F. (2016). Predictores del miedo y las habilidades sociales en adolescentes del contexto pluricultural de Ceuta. Acción Psicológica, 13(1), 179-190. https://doi.org/10.5944/AP.13.1.15560

Ramírez, G.M., Collazos, C.A., Moreira, F., \& Fardoun, H. (2018). Relación entre el U-Learning, aprendizaje conectivo y el estándar xAPI: Revisión Sistemática. Campus Virtuales, 7(1), 51-62. https://bit.ly/3gU8qXm

Reis, H.T., \& Gable, S.L. (2013). Toward a positive psychology of relationships. In C. L. Keyes \& J. Haidt (Eds.). Flourishing: The positive person and the good life (pp. 129-159). American Psychological Association.

Richardson, W.S., Wilson, M.C., Nishikawa, J., \& Hayward, R.S. (1995). The well-built clinical question: a key to evidencebased decisions. ACP Journal Club, 123(3), A12-A13. http://doi.org/10.7326/ACPJC-1995-123-3-A12

Salavera, C., Usán, P., \& Teruel, P. (2019). The relationship of internalizing problems with emotional intelligence and social skills in secondary education students: gender differences. Psicologia: Reflexao e Critica, 32, 4. https://doi.org/10.1186/s41155-018-0115-y

Sali, G. (2019). Examining the development of creativity in adolescents in 9th and 12th grades: A four-year longitudinal study. Creativity studies, 12(4), 341-360. https://doi.org/10.3846/cs.2019.10260

Sánchez-Escobedo, P., Misiūnienè, J., \& Mockaitytè, E. (2015). A comparison of non-verbal creativity between Lithuanian and Mexican adolescents. International Journal of Psychology: A Biopsychosocial Approach / Tarptautinis Psichilogijos Žurnalas: Biopsichosocialinis Požiūris, 17, 25-38. http://dx.doi.org/10.7220/2345-024X.17.2

Sánchez-Hernando, B., Juárez-Vela, R., Antón-Solanas, I., Gasch-Gallén, Á., Melo, P., Nguyen,T.H., Martínez-Riera, J.R., Ferrer-Gracia, E., \& Gea-Caballero, V. (2021). Association between Life Skills andAcademic Performance in Adolescents in the Autonomous Community of Aragon (Spain). International Journal of Environmental Research Public, 18(8), 4288. https://doi.org/10.3390/ijerph18084288

Sánchez-Meca, J. (2010). Cómo realizar una revisión sistemática y un meta-análisis. Aula abierta, 38(2), 53-64. https://doi.org/10.17811/rifie.38.1,\%202.2010

Sastre-Riba, S., \& Pascual-Sufrate, M.T. (2013). Alta capacidad intelectual, resolución de problemas y creatividad. Revista de Neurología, 56(1), 67-76. https://doi.org/10.33588/rn.56S01.2013025

Schenk, L., Sentse, M., Lenkens, M., Nagelhout, G.E., Engbersen, G., \& Severiens, S. (2020). An Examination of the Role of Mentees' Social Skills and Relationship Quality in a School-Based Mentoring Program. American Journal of Community Psychology, 65(1-2), 149-159. https://doi.org/10.1002/ajcp.12397

Sosa, S.S., \& Salas-Blas, E. (2020). Resiliencia y habilidades sociales en estudiantes secundarios de San Luis de Shuaro, La Merced. Revista Comuni@cción, 11(1), 40-50. https://doi.org/10.33595/2226-1478.11.1.394

Suarez-López, A., \& Ramos-Noboa, I. (2018). La intervención psicológica como estrategia de desarrollo cognitivoconductual en los adolescentes con retraso mental y deficiencias sociales. Revista Ciencia \& Tecnología, 18(17), 156-171. https://doi.org/10.47189/rcct.v18i17.161

Tacca, D.R., Cuarez, R., \& Quispe, R. (2020). Habilidades Sociales, Autoconcepto y Autoestima en Adolescentes Peruanos de Educación Secundaria, International Journal of Sociology of Education, 9(3), 293-324 http://doi.org/10.17583/rise.2020.5186

Taja, V.A., Tej, J., \& Sirkova, M. (2015). Creative management techniques and methods as a part of the management education: analytical study on students' perceptions. Procedia. Social and Behavioral Sciences, 197, 1918-1925. https://doi.org/10.1016/j. sbspro.2015.07.563

Tajer, D.J., Reid, G.B., Cuadra, M.E., Solis, M., Fernández, J., Saavedra, L.D., Lavarello, M.L., \& Fabbio, R.P. (2019). Varones adolescentes en la Ciudad de Buenos Aires: barreras de género en la prevención y atención de la salud. Revista Salud Colectiva, 15, e2256. https://doi.org/10.18294/sc.2019.2256

Urrútia, G., \& Bonfill, X. (2010). Declaración PRISMA: una propuesta para mejorar la publicación de revisiones sistemáticas y metaanálisis. Medicina clínica, 135(11), 507-511. https://doi.org/10.1016/i.medcli.2010.01.015

Viejo, C., \& Ortega-Ruiz, R. (2015). Cambios y riesgos asociados a la adolescencia. Psychology, Society \& Education, 7(2), 109-118. http://dx.doi.org/10.25115/psye.v7i2.527

Von Hohendorff, J., de Paula Couto, M. C. P., \& Eschiletti, L. E. (2013). Social skills in adolescence: Psychopathology and sociodemographic variables. Estudos de Psicologia, 30(2), 151-160. https://doi.org/10.1590/S0103166X2013000200001

Vorobjov, S., Saat, H., \& Kull, M. (2014). Social skills and their relationship to drug use among 15-16-year-old students in Estonia: An analysis based on the ESPAD data. Nordic Studies on Alcohol and Drugs, 31(4), 401-412. https://doi.org/10.2478/nsad-2014-0031

Zegarra, C.O., \& Cuba, M. (2017). Frecuencia de adicción a Internet y desarrollo de habilidades sociales en adolescentes de una zona urbana de Lima. Revista Biomédica Medwave, 17(1), e6857. https://doi.org/10.5867/medwave.2017.01.6857 